\title{
Banco de Dentes Humanos: ética a serviço do ensino e da pesquisa - a experiência da Faculdade de Odontologia da UERJ
}

\author{
Human tooth bank: ethics in the service of teaching and research - the \\ experience of UERJ School of Dentistry
}

Leonardo Navega Louzada ${ }^{1}$, Roberta Costa Jorge ${ }^{2}$, Kelly dos Santos Silva ${ }^{3}$, Rafael dos Santos Lemos Pacífico $^{4}$, Felipe Fernandes de Paula Dantas ${ }^{5}$, Stephanie Evellyn Antunes Novaes ${ }^{6}$, Izabel Monteiro D’Hyppolito $^{7}$, Luana da Silva Viana ${ }^{8}$, Byanca Ramos de O. Correia ${ }^{9}$, Beatriz Farias do Nascimento ${ }^{10}$, Gabriela C. Andrade Americano ${ }^{11}$, Vera Mendes Soviero ${ }^{12}$

\begin{abstract}
1 Aluno de graduação. Universidade do Estado do Rio de Janeiro (UERJ), Brasil. E-mail: leonardolouzada9@gmail.com 2 Aluna de graduação. Universidade do Estado do Rio de Janeiro (UERJ), Brasil. E-mail: robertacjorge@gmail.com 3 Aluna de graduação. Universidade do Estado do Rio de Janeiro (UERJ), Brasil. E-mail: kelly.odontouerj@gmail.com 4 Aluno de graduação. Universidade do Estado do Rio de Janeiro (UERJ), Brasil. E-mail: pacifico.uerj@gmail.com 5 Aluno de graduação. Universidade do Estado do Rio de Janeiro (UERJ), Brasil. E-mail: felipedantas.bh@gmail.com 6 Aluna de graduação. Universidade do Estado do Rio de Janeiro (UERJ), Brasil. E-mail: fanynovaes@gmail.com 7 Aluna de graduação. Universidade do Estado do Rio de Janeiro (UERJ), Brasil. E-mail: izabelmdh@gmail.com 8 Aluna de graduação. Universidade do Estado do Rio de Janeiro (UERJ), Brasil. E-mail: luana-sviana@hotmail.com 9 Aluna de graduação. Universidade do Estado do Rio de Janeiro (UERJ), Brasil. E-mail: byaramoscorreia@gmail.com 10 Aluna de graduação. Universidade do Estado do Rio de Janeiro (UERJ), Brasil. E-mail: beatriz_farias@yahoo.com.br 11 Bolsista Qualitec. Universidade do Estado do Rio de Janeiro (UERJ), Brasil. E-mail: americanogabriela@gmail.com 12 Professora adjunta. Universidade do Estado do Rio de Janeiro (UERJ), Brasil. E-mail: verasoviero@gmail.com
\end{abstract}

Recebido em: 11/02/2015 | Aprovado em: 08/07/2015

DOI: $10.12957 /$ interag.2015.21857

\section{Resumo}

A utilização de dentes humanos é rotineira na formação profissional e em pesquisas científicas de alta relevância em Odontologia. Um banco de dentes humanos permite que atividades didático-científicas que utilizam dentes humanos sejam realizadas dentro dos preceitos éticos quanto ao uso de material biológico, contribuindo para coibir o comércio de dentes humanos. $O$ objetivo do presente artigo foi abordar questões éticas e legais acerca do emprego de dentes humanos no ensino e na pesquisa em Odontologia e relatar a experiência da Faculdade de Odontologia da UERJ com o projeto Banco de Dentes Humanos, implantado como um projeto de extensão, com caráter multidisciplinar, resultante do trabalho dos estudantes vinculados ao Programa de Educação Tutorial (PET), sob a orientação do professor tutor. O BDH FO-UERJ reforça a indissociabilidade da tríade ensino-pesquisa-extensão e tem tido importante função na formação ética dos estudantes de Odontologia, no suporte às atividades didáticocientíficas e no estímulo à discussão do tema junto à sociedade.

Palavras-chave: Dentes humanos; Educação; Ética.

Área temática: Saúde.

Linha de extensão: Saúde humana.

\begin{abstract}
Human teeth are routinely used in dental education and in relevant research in the field of dentistry. With the support of a tooth bank, educational activities and scientific experiments that depend on natural human teeth are carried out following the ethical guidelines regarding the use of human biological material. Besides, illegal acquisition of human teeth is discouraged. The aim of the present paper is to review ethical and legal aspects of the utilization of natural human teeth in dental education and research and to report the experience of the Tooth Bank Project at the School of Dentistry at the University of the State of Rio de Janeiro, which was set up as a community project. A group of undergraduate students who are linked to the Tutorial Educational Program (TEP) has participated in the implementation and routine of the tooth bank under the guidance of the tutor. The Tooth Bank reinforces the inseparable triad of education-research-community and has played an important role both in ethics education in dentistry and in supporting dental education, research and community activities.
\end{abstract}

Keywords: Human teeth; Education; Ethics.

\section{Introdução}


O ensino da anatomia humana que se utiliza de cadáveres e órgãos humanos não somente na Odontologia, mas nos diversos cursos das ciências da saúde, é bastante conhecido. As especificidades da estrutura dos tecidos dentários requerem, na Odontologia, que dentes humanos também sejam disponibilizados para atividades didático-pedagógicas. $\mathrm{O}$ emprego de dentes humanos para o estudo da anatomia e treinamento pré-clínico é bem antigo na Odontologia, sendo essencial para a formação profissional $^{1}$. A utilização de dentes humanos em pesquisa científica também é bastante frequente. Inúmeras pesquisas científicas de alta relevância para o avanço do diagnóstico e tratamento de agravos da saúde bucal dependem do emprego de dentes humanos em seus experimentos. Os estudos avaliam, em dentes humanos, características morfológicas e bioquímicas dos tecidos dentários, além de testarem materiais odontológicos utilizados na clínica ${ }^{2}$.

Inúmeras discussões acerca de questões morais e éticas sobre o emprego de cadáveres para fins de estudo ou pesquisa científica resultaram na Lei no $8 .{ }^{16} 01$, de $1992^{3}$, que dispõe sobre a utilização de cadáver para estudo ou pesquisa.

Já em relação aos dentes humanos, durante muito tempo, nenhuma legislação ou recomendação oficial de órgãos governamentais direcionou o seu emprego, seja para atividades de ensino ou de pesquisa científica. De certo modo, sempre houve um consenso de que a obtenção de dentes indicados para extração, por exemplo, deveria ocorrer mediante autorização do paciente. Entretanto, a formalização dessa autorização por meio da assinatura de um termo de consentimento não era habitual. Ainda hoje, não é raro encontrarmos potes de coleta de dentes extraídos em locais de atendimento odontológico de urgência, sem que exista qualquer padronização quanto ao modo de obtenção desses dentes ou formalização do consentimento dos pacientes.

Mais preocupante é o fato de alguns cursos de Odontologia solicitarem dentes humanos aos estudantes para as práticas de ensino, sem se preocuparem com o modo como tais dentes são obtidos. Estudantes de Odontologia relataram ter comprado dentes para 
atender às necessidades da prática acadêmica, ${ }^{4,5}$, cientes ou não, de que estavam incorrendo em crime de comercialização de órgãos ${ }^{6}$.

\section{Objetivo}

O objetivo do presente artigo foi abordar questões éticas e legais acerca do emprego de dentes humanos no ensino e na pesquisa em Odontologia e relatar a experiência da Faculdade de Odontologia da UERJ com o projeto Banco de Dentes Humanos.

\section{Aspectos éticos e legais}

A utilização de dentes humanos, assim como de demais órgãos do corpo humano, está sujeita às legislações vigentes que dispõem sobre a matéria. Inicialmente tratada pela Lei no 4.280 de 6 de novembro de $1963^{7}$, que dispôs sobre a extirpação de órgãos ou tecidos de pessoas falecidas, atualmente segue as disposições da Lei no 9.434 de 4 de fevereiro de $1997^{6}$ sobre a retirada de órgãos e partes do corpo humano, em vida ou post mortem, para fins de transplantes e tratamento e outras providências. Incorre em crime e fica sujeito às sanções penais, aquele que remover, transportar, guardar ou distribuir tecidos, órgãos ou partes do corpo humano de pessoa ou cadáver que tenham sido obtidos em desacordo com dispositivos da referida lei.

Para fins de pesquisa científica, a matéria foi mais amplamente tratada pela Resolução CNS no 196 de 10 de outubro de $1996^{8}$, revogada recentemente pela Resolução CNS no 466 de 12 de dezembro de $2012^{9}$ que dispõe sobre diretrizes e normas regulamentadoras de pesquisas envolvendo seres humanos.

Especificamente sobre o armazenamento e emprego de material biológico humano, no âmbito de projetos de pesquisa, o Conselho Nacional de Saúde aprovou, em 2005, a Resolução no $347^{10}$ que foi posteriormente revogada pela Resolução no 441 de 12 de maio de $2011^{11}$, determinando que material biológico com finalidade de pesquisa deve ser captado e armazenado em biobancos institucionalizados. De acordo com esta resolução, a 
utilização de material biológico humano em pesquisa fica condicionada à constituição e ao funcionamento de um biobanco de material biológico humano, regulamentado e aprovado pelo comitê de ética institucional e com parecer final da CONEP. No mesmo ano, a Portaria Ministerial no $2.201^{12}$ estabeleceu diretrizes éticas e legais para a implantação e funcionamento de biobancos e biorrepositórios de material biológico humano.

A implantação de bancos de dentes humanos é prevista no documento da ANVISA sobre prevenção e controle de riscos em Odontologia ${ }^{13}$ como um meio de criar mecanismos para coibir a aquisição de dentes humanos por meios ilegais. O capítulo XIV do Código de Ética Odontológico ${ }^{14}$, que dispõe sobre doação, transplante e banco de órgãos, tecido e biomateriais, esclarece que o profissional que descumprir à legislação vigente sobre o assunto, incorre em infração ética e fica sujeito às penas previstas na Lei no 4.324 de 14 de abril de $1964^{15}$ que preveem desde a advertência ou censura até a suspensão ou cassação do exercício profissional.

Sendo assim, não somente por questões morais e éticas, mas também do ponto de vista legal, fica clara a necessidade da implantação de bancos de dentes humanos por instituições de ensino e pesquisa em Odontologia que venham a atender às suas necessidades didático-científicas. No Brasil, a Faculdade de Odontologia da Universidade de São Paulo foi pioneira na implantação de um Banco de Dentes Humanos e na divulgação de programas e campanhas de doação de dentes decíduos na década de $80^{16}$. A partir de então, outras instituições, das diferentes regiões do país, desenvolveram projetos e implantaram bancos de dentes ${ }^{1,17}$ e o tema passou a ser mais amplamente discutido, além de questões éticas e metodológicas do emprego de dentes humanos no ensino e na pesquisa odontológica passaram a ser alvo de inúmeras pesquisas ${ }^{18}$.

\section{A experiência do Banco de Dentes Humanos da Faculdade de Odontologia da UERJ (BDH FO-UERJ)}


A implantação e a condução das atividades do BDH FO-UERJ tiveram e têm participação ativa dos estudantes de graduação em Odontologia integrantes do Programa de Educação Tutorial (PET) do Ministério da Educação. Desde a fase de planejamento, sempre houve um entendimento claro de que as atividades envolvidas na implementação e condução de um banco de dentes traziam, na sua essência, conceitos e práticas da Extensão Universitária. Por isso, na FO-UERJ, o banco de dentes constituiu-se como um Projeto de Extensão cadastrado na Sub-reitoria de Extensão da UERJ. No âmbito da FO, o BDH conta com o respaldo da anuência e suporte da Direção.

Através da implementação e condução do banco de dentes na FO-UERJ, temos tido a oportunidade de trabalhar questões altamente relevantes para o ensino, para a pesquisa e para a extensão, sendo extremamente benéfica a participação ativa de estudantes nesse processo. De acordo com o documento da Política Nacional de Extensão Universitária ${ }^{19}$, a Extensão Universitária é um processo educativo, cultural, científico e político que articula o ensino e a pesquisa de forma indissociável e viabiliza uma relação transformadora entre universidade e sociedade, em uma via de mão dupla, em que a universidade influencia e também é influenciada pela comunidade.

Nesse contexto, são infinitas as possibilidades de se trabalhar a extensão em sua plenitude através de um banco de dentes, levando-se em consideração o arsenal de questões éticas, morais, científicas e políticas envolvidas em todo o processo. Afinal, um exemplo dessas possibilidades é fato do projeto oferecer suporte a atividades de ensino e pesquisa em Odontologia que somente são possíveis porque a sociedade é sensibilizada através de ações do próprio projeto. Ao envolver a sociedade, tem-se a oportunidade de se discutir questões como doação de órgãos e legislações que regulamentam o emprego de material biológico em pesquisas com a comunidade acadêmica e com a sociedade como um todo. O BDH FO-UERJ, além das atividades operacionais de captação, limpeza, catalogação e armazenamento de dentes humanos, oferece suporte a atividades de didático-científicas e desenvolve ações de conscientização quanto ao emprego de dentes humanos no ensino e na pesquisa. 


\section{Ações de Conscientização}

O BDH FO-UERJ realiza ações de conscientização junto ao público interno e externo à FO-UERJ. No âmbito da FO-UERJ, as atividades são direcionadas ao corpo docente, discente e técnico-administrativo, com foco principal para as disciplinas que mais desenvolvem atividades de ensino e pesquisa com dentes humanos.

Discussões em sala de aula e em fóruns que reúnam integrantes do corpo docente, discente e/ou técnico-administrativo vêm sendo promovidas, no sentido de se explorar questões éticas, legais e científicas relacionadas ao tema. Os pacientes que buscam atendimento nas Clínicas Odontológicas de Ensino da FO têm a oportunidade de terem contato com o tema através de material educativo exposto em murais e esclarecimentos quanto à possibilidade de cederem seus dentes que porventura tenham indicação de extração.

O projeto também participa de eventos com a participação da comunidade externa, como a Semana da UERJ sem Muros. Prova de que o tema vem despertando cada vez mais interesse da sociedade é o fato de que o BDH FO-UERJ foi contatado por três emissoras de rádios, sendo elas a CBN, a Rádio-Relógio e a Rádio UERJ, e concedeu entrevistas visando esclarecer dúvidas sobre o tema, aproximando-se, cada vez mais, da sociedade que o cerca.

\section{Captação de Dentes}

A principal fonte de captação de dentes ainda são as próprias Clínicas Odontológicas de Ensino da FO-UERJ. Caixas de coleta, com potes individuais contendo água destilada e termos de consentimento livre e esclarecido (TCLE), são disponibilizados para as clínicas. Quando um paciente tem indicação de extração de um dente, ele é informado sobre a possibilidade de ceder seu dente para o BDH FO-UERJ. Havendo interesse do paciente, o TCLE é lido, esclarecido e assinado. Após a extração, o dente é acondicionado em um dos potes com água destilada que será identificado com o número do TCLE correspondente. $\mathrm{O} \mathrm{BDH}$ tem hoje, na Disciplina de Cirurgia Bucal, sua maior fonte de 
captação, seguida pela Clínica Integrada e pela Odontopediatria. Visando ampliar a captação de dentes, o BDH FO-UERJ vem realizando contato com outras unidades de serviço odontológico de instituições públicas que tenham interesse em se tornar parceiras do projeto.

O BDH FO-UERJ também pode receber dentes diretamente de pessoas que, porventura, tenham seus dentes, ou de seus filhos, guardados em casa. Nesse caso, o interessado deve entrar com contato por telefone, e-mail ou pessoalmente para ser informado e esclarecido sobre o processo.

\section{Limpeza e Catalogação}

No BDH, os dentes coletados são limpos de remanescentes de tecidos moles e/ou cálculo dentário, utilizando curetas manuais e aparelho de ultrassom. Em seguida, são catalogados, numerados individualmente e armazenados em novos potes individuais contendo água destilada, em geladeira.

Dentes solicitados para atividades de ensino passam por processo de esterilização, mais comumente por esterilização em autoclave, ou esterilização química se o dente apresentar restauração de amálgama ${ }^{20,21}$. Dentes solicitados para atividades de pesquisa não são esterilizados no BDH. O pesquisador solicitante é informado de que a esterilização dos dentes fica a cargo do pesquisador, pois a definição do método de esterilização a ser usado depende do tipo de experimento que será realizado. Todas as etapas do processo seguem Procedimentos Operacionais Padrão (POPs) específicos.

Para a catalogação, os dentes são classificados por tipo, características anatômicas e pela presença de eventuais alterações de desenvolvimento e/ou adquiridas. Informações relativas ao paciente, como sexo e idade também são registradas. Os dados de classificação são armazenados em banco de dados digital (elaborado no programa EpiInfoTM 7, CDC, USA), facilitando, posteriormente, a seleção de dentes com características específicas dependendo do tipo de atividade para a qual os dentes estiverem sendo solicitados. 
Visando ampliar as possibilidades de apoio a atividades didático-pedagógicas, o BDH FOUERJ vem desenvolvendo um banco de imagens, para o qual todos os dentes coletados são fotografados.

\section{Transferência de dentes para atividades didático-científicas}

A solicitação e transferência de dentes humanos para atividades didático-científicas são feitas mediante o preenchimento de formulários próprios. O BDH FO-UERJ vem oferecendo suporte às atividades de disciplinas curriculares do curso de graduação em Odontologia, como Endodontia, Prótese Fixa, Prótese Removível, Dentística e Odontopediatria.

O BDH FO-UERJ segue as recomendações da legislação pertinente no que diz respeito ao seu funcionamento como biobanco de modo a oferecer suporte também a atividades de pesquisa. Pelo Parecer CONEP (BIOBANCO) no 014/2015, datado de 31/03/2015, o BDH FO-UERJ foi aprovado como biobanco de dentes humanos.

\section{O BDH FO-UERJ como instrumento de integração ensino-pesquisa- extensão}

O trabalho de conscientização sobre o uso apropriado de material biológico humano no ensino e na pesquisa em Odontologia tem tido um impacto significativo na formação ética e cidadã dos estudantes. Na FO-UERJ, discussões sobre a legislação pertinente e questões morais relacionadas ao tema vêm à tona em função da existência do BDH e acabam por gerar um impacto em todo o corpo discente. Além disso, o laboratório do BDH FO-UERJ constitui-se em um cenário de prática de atividades específicas, tais como: reconhecimento de características anatômicas normais e patológicas do dentes; aplicação de procedimentos de limpeza dos dentes usando instrumentos odontológicos manuais e ultrassônicos; prática de documentação fotográfica; prática com software de banco de dados e análises estatísticas; dentre outras. Sendo assim, inúmeros conteúdos específicos e não específicos do curso de Odontologia são trabalhados com os estudantes, tendo o BDH como eixo de integração. 
Além de dar suporte a atividades de pesquisa, o BDH FO-UERJ constitui-se em um laboratório que tem potencial e suas próprias demandas de pesquisa. Ainda são muitos os questionamentos acerca de metodologias que envolvem o emprego de dentes humanos para os quais não se tem as respostas. Desde questões mais simples como encontrar um método mais eficiente de limpeza dos dentes até questões mais complexas como definir o melhor método de armazenamento que viabilize a extração tardia de células-tronco da polpa dentária, por exemplo. Pensando nas inúmeras possibilidades que podem advir de tais questionamentos, em meados de 2014, o BDH FO-UERJ foi cadastrado como uma Unidade de Desenvolvimento Tecnológico (UDT) junto ao Departamento de Inovação da UERJ (InovUERJ).

À medida em que o projeto trabalha de modo simultâneo e indissociável questões éticas, morais, didáticas e científicas, a interface com a sociedade torna-se, ao mesmo tempo, um propósito e uma consequência. Afinal, as demandas surgem não somente da comunidade acadêmica, mas também da sociedade como um todo que anseia por atitudes e inovações que possam trazer benefícios para todos.

\section{Discussão}

A importância dos bancos de dentes para dar suporte às atividades didático-científicas dos cursos de Odontologia é inquestionável. A utilização de dentes humanos é essencial para a formação acadêmica em Odontologia e, se não são criados mecanismos para a obtenção adequada dos mesmos, permite-se a permanência de uma lacuna importante na formação ética dos estudantes. Mais do que isso, incorre-se em descumprimento da legislação vigente sobre a matéria ${ }^{6}$.

O modo pelo qual os cursos de Odontologia se organizam para implementar um banco de dentes varia de acordo com as condições e propostas de cada instituição, ${ }^{1,17}$. Pioneiros na implantação de um banco de dentes e na regulamentação do uso de dentes humanos no ensino e na pesquisa, o Banco de Dentes da Faculdade de Odontologia da Universidade de São Paulo é um modelo para a comunidade acadêmico-científica 
odontológica $^{16}$. Adaptações às condições de infraestrutura e demais recursos de cada instituição são necessárias, desde que respeitadas as legislações vigentes ${ }^{18}$.

A opção da FO-UERJ de fazê-lo no formato de um projeto de extensão, com participação ativa dos estudantes em todas as atividades relacionadas ao banco de dentes, atende às propostas didático-pedagógicas do curso, cria um cenário de práticas pedagógicas, favorece a integração graduação/pós-graduação, além de contribuir para o fortalecimento da extensão na FO com um projeto que apresenta múltiplas vertentes e que lida com questões que instigam discussões com a sociedade.

Independentemente do caminho escolhido por cada instituição para assegurar que dentes humanos sejam obtidos e empregados dentro dos preceitos éticos e legais adequados, é importante que a estratégia selecionada una corpo docente, discente e técnicoadministrativo em torno de propósitos comuns, fortalecendo questões conceituais e estimulando atitudes de coerência entre todos os setores. A experiência da FO-UERJ tem nos mostrado que a construção coletiva do projeto tem sido um facilitador nesse sentido. Principalmente pela participação ativa de estudantes que se encontram em diferentes estágios do curso de graduação, e acabam por atuar como multiplicadores dos conceitos e atitudes do projeto juntos aos seus colegas, professores e demais setores da faculdade.

O BDH FO-UERJ tem a intenção de ampliar a captação através de outras fontes de captação de dentes além da FO-UERJ. Para tal, contatos com instituições públicas e privadas que realizam assistência odontológica vêm sendo realizados no sentido de se multiplicar a captação de dentes para além dos limites da FO-UERJ.

Para atingir a importante missão de desencorajar a prática de comércio de dentes, que envolve não apenas dentes extraídos in vivo, mas, principalmente, a violação de cadáveres, os bancos de dentes trabalham em torno da motivação dos pacientes para a doação, cuja colaboração é fundamental. Por isso, não se pode deixar de mencionar a participação dos pacientes que se sensibilizam e entendem que seus dentes podem ser muito mais de que 
"pequenas joias" guardadas em caixinhas no fundo de alguma gaveta, podendo representar uma significativa contribuição para o ensino e a pesquisa.

\section{Considerações finais}

O BDH FO-UERJ tem viabilizado que atividades didático-científicas que utilizam dentes humanos sejam realizadas dentro dos preceitos éticos que norteiam a utilização de material biológico. Além disso, atua no esclarecimento, conscientização e motivação para a doação de dentes junto à sociedade.

\section{Agradecimentos}

Ao Ministério da Educação que provê bolsas de iniciação científica para os estudantes de graduação vinculados ao Programa de Educação Tutorial. Ao Departamento de Inovação da UERJ que proporciona uma Bolsa Qualitec para um profissional nível Mestrado vinculado ao projeto.

\section{Conflito de Interesse}

Os autores declaram não haver nenhum conflito de interesse.

\section{Referências}

1. FREITAS, A.B.D.A.; PINTO, S.L.; TAVARES, E.P.; BARROS, L.M.; CASTRO, C.D.L.; MAGALHÃES, C.S. Uso de Dentes Humanos Extraídos e os Bancos de Dentes nas Instituições Brasileiras de Ensino de Odontologia. Pesq Bras Odontoped Clin Integr, v.12, n.1, p.59-64, jan./mar., 2012.

2. FREITAS, A.B.D.A.; CASTRO, C.D.L.; SETT, G.S.J.; BARROS, L.M.; MOREIRA, A.N.; MARGALHÃES, C.S. Uso de dentes extraídos nas pesquisas odontológica publicadas em periódicos brasileiros de acesso online gratuito: um estudo sob o prisma da bioética. Arquivos em Odontologia, v.46, n.3, p. $\mathrm{jul} / \mathrm{set} 2010$.

3. BRASIL. Lei $\mathrm{n}^{\circ} 8.501$ de 30 de novembro de 1992. Disponível em: http://www.planalto.gov.br/ccivil_03/leis/L8501.htm. 
4. PAUlA, S.; BITTENCOURT, L.P.; PIMENTEL, E.; GABRIEL FILHO, P.A.; IMPARATO, J.C.P. Comercialização de dentes na universidades. Pesq Bras Odontoped Clin Integr, v.1, n.3, p.38-41, 2001.

5. COSTA, S.M.; MAMELUQUE, S.; BRANDÃO, E.L.; MELO, A.E.M.A.; PIRES, C.P.A.B.; REZENDE, E.J.C.; ALVES, K.M. Dentes humanos no ensino odontológico: procedência, utilização, descontaminação e armazenamento pelos acadêmico de UNIMONTES. Revista da ABENO, v.7, n.1, p.6-12, jan/abr 2007.

6. BRASIL. Lei $\mathrm{n}^{\circ} 9.434$ de 4 de fevereiro de 1997. Disponível em: http://www.planalto.gov.br/ccivil_03/leis/19434.htm

7. BRASIL. Lei $\mathrm{n}^{\circ} 4.280$ de 6 de novembro de 1963. Disponível em: http://www.lexml.gov.br/urn/urn:lex:br:federal:lei:1963-11-06;4280

8. BRASIL. Resolução CNS no 196 de 10 de outubro de 1996. Disponível em: http://conselho.saude.gov.br/web_comissoes/conep/aquivos/resolucoes/23_out_vers ao_final_196_ENCEP2012.pdf

9. BRASIL. Resolução CNS $n^{0} 441$ de 12 de maio de 2011. Disponível em: http://conselho.saude.gov.br/resolucoes/2011/Reso441.pdf

10. BRASIL. Resolução CNS n 347 de 13 de janeiro de 2005. Disponível em: http://conselho.saude.gov.br/web_comissoes/conep/aquivos/resolucoes/resolucoes.h tm

11. BRASIL. Resolução CNS no 411 de 12 de maio de 2011. Disponível em: http://conselho.saude.gov.br/resolucoes/2011/Reso441.pdf

12. BRASIL. Portaria MS no 2.201 de 14 de setembro de 2011. Disponível em: http://bvsms.saude.gov.br/bvs/saudelegis/gm/2011/prt2201_14_09_2011.html

13. BRASIL. Ministério da Saúde. Agência Nacional de Vigilância Sanitária. Serviços Odontológicos: prevenção e controle de riscos. Brasília: Ministério da Saúde, 2006. $156 \mathrm{p}$.

14. CFO. Código de Ética Odontológico. Resolução CFO nº 118/2012. Disponível em: http://cfo.org.br/wp-content/uploads/2009/09/codigo_etica.pdf

15. BRASIL. Lei $\mathrm{n}^{\circ} 4.324$ de 14 de abril e 1964. Disponível em: http://www.planalto.gov.br/ccivil_03/leis/1950-1969/L4324.htm

16. IMPARATO, J.C.P. et al. Banco de Dentes Humanos. 20 ed. Curitiba: Maio, 2003. 189p apud PEREIRA, D.Q. Banco de dentes humanos no Brasil: revisão de literatura. Revista da ABENO, v.12, n.2, p.178-184, 2012.

17. MOREIRA, L.; GENARI, B.; STELlO, R.; COLlARES, F.M.; SAMUEL, S.M.W. Banco de dentes humanos para o ensino e pesquisa em Odontologia. Rev Fac Odontol Porto Alegre, v.50, n.1, p.34-37, jan/abr, 2009. 
18. PEREIRA, D.Q. Banco de dentes humanos no Brasil: revisão de literatura. Revista da ABENO, v.12, n.2, p.178-184, 2012.

19. BRASIL. Política Nacional de Extensão Universitária. Fórum de Pró-reitores de Extensão das Universidades Públicas Brasileiras. 2012. Disponível em: http://www.renex.org.br/documentos/2012-07-13-Politica-Nacional-de-Extensao.pdf

20. DOMINICI, J.T.; ELEAZER, P.D.; CLARK, S.J.; STAAT, R.H.; SCHEETZ, J.P. Disinfection/sterilization of extracted teeth for dental student use. J Dent Educ, v.65, n.11, p.1278-80, 2001.

21. KUMAR, M.; SEQUEIRA, P.S.; PETER, S.; BHAT, G.K. Sterilisation of extracted human teeth for educational use. Indian J Med Microbiol, v.23, n.4, p.2568, 2005. 